University of South Florida

DIGITAL COMMONS

Digital Commons @ University of

@ UNIVERSITY OF SOUTH FLORIDA

South Florida

$1-1-2015$

\title{
2015 Work Plan USF Sarasota-Manatee
}

USF

Follow this and additional works at: https://digitalcommons.usf.edu/usf_accountability_reports

\section{Scholar Commons Citation}

USF, "2015 Work Plan USF Sarasota-Manatee" (2015). USF Accountability Reports. 53.

https://digitalcommons.usf.edu/usf_accountability_reports/53

This Article is brought to you for free and open access by the USF Archives at Digital Commons @ University of South Florida. It has been accepted for inclusion in USF Accountability Reports by an authorized administrator of Digital Commons @ University of South Florida. For more information, please contact digitalcommons@usf.edu. 


\section{INTRODUCTION}

The State University System of Florida has developed three tools that aid in guiding the System's future.

1) The Board of Governors' 2025 System Strategic Plan is driven by goals and associated metrics that stake out where the System is headed;

2) The Board's Annual Accountability Report provides yearly tracking for how the System is progressing toward its goals;

3) Institutional Work Plans connect the two and create an opportunity for greater dialogue relative to how each institution contributes to the System's overall vision.

These three documents assist the Board with strategic planning and with setting short-, mid- and long-term goals. They also enhance the System's commitment to accountability and driving improvements in three primary areas of focus: 1) academic quality, 2) operational efficiency, and 3) return on investment.

The Board will use these documents to help advocate for all System institutions and foster even greater coordination with the institutions and their Boards of Trustees.

Once a Work Plan is approved by each institution's respective Boards of Trustees, the Board of Governors will review and consider the plan for potential acceptance of 2015-16 components. Longer-term components will inform future agendas of the Board's Strategic Planning Committee. The Board's acceptance of a work plan does not constitute approval of any particular component, nor does it supersede any necessary approval processes that may be required for each component. 


\section{TABLE OF CONTENTS}

\section{STRATEGY}
a. Mission Statement
b. Vision Statement
c. Statement of Strategy
d. Strengths and Opportunities
e. Key Initiatives \& Investments

\section{PERFORMANCE BASED FUNDING METRICS}

\section{OTHER KEY PERFORMANCE INDICATORS}
a. Goals Common to All Universities
b. Goals Specific to Research Universities
c. Institution Specific Goals

\section{OPERATIONS}
a. Fiscal Information
b. Enrollment Planning
c. Academic Program Coordination

\section{DEFINITIONS}




\section{MSSION STATEMENT* (What is your purpose?)}

The University of South Florida Sarasota-Manatee (USFSM) provides high-quality bachelor's and masters-level education and scholarly activity in a personalized learning community that prepares successful leaders and responsible citizens.

\section{VSION STATEMENT* (What do you aspire to?)}

As a valued member of the USF System, USFSM will be nationally recognized as a student-centered, research focused, community-engaged university with significant economic and cultural impact to the region.

\section{STATEMENT OF STRATEGY (How will you get there?)}

Given your mission, vision, strengths and available resources, provide a brief description of your market and your strategy for addressing and leading it.

USFSM's target markets include Florida College System transfers, returning adult students, and local area high school graduates who wish to stay within their community while pursuing a public university education. USFSM serves this market by offering individualized attention through a low (13-to-1) student-faculty ratio. The institution provides a variety of course delivery options and class schedules to meet the needs of a diverse student population. Additionally, USFSM benefits from the many shared resources available as a valued a member of the USF System. 


\section{STRENGTHS AND OPPORTUNTIES (within 3 years)}

What are your core capabilities, opportunities and challenges for improvement?

USF Sarasota-Manatee is privileged to provide education that permits students to "learn where they earn." The institution capitalizes on its low student-to-faculty ratio by offering individualized attention to ensure that students are supported, retained, and successful. As a member of the USF System, USF Sarasota-Manatee benefits from the efficiency of shared resources, the opportunity for collaboration with other member institutions, and a unified brand that yields identity and impact. Over the next three years, the institution's greatest opportunities are to grow lower-level and STEM programming.

\section{KEY INITIATIVES \& INVESTMENTS (within 3 years)}

Describe your top three key initiatives for the next three years that will drive improvement in Academic Quality, Operational Efficiency, and Return on Investment.

1. Implement USFSM Strategic Plan 2015-2020: During the spring of 2015, USF Sarasota-Manatee and its community partners formulated a new five-year strategic plan. The plan emphasizes student success, increased campus life for students, increased enrollment, quality teaching and learning, scholarly activity, community engagement, and resource development. The plan is expected to be completed by the fall of 2015.

2. Expand STEM programs: USF Sarasota-Manatee's B.S. in Biology program, delivered through a partnership with Mote Marine Laboratory, enrolled its first class in fall 2014. In 2014-15, 100 students chose Biology as a major, demonstrating high demand for science programs in the region. Key initiatives to grow the STEM programming include hiring additional faculty and expanding laboratory and research space.

3. Preserve, grow, and diversify the financial, private, and other resources: USF Sarasota-Manatee will strategically increase efforts in grant writing, private fundraising, and developing public-private partnerships. Additionally, through planned enrollment increases the institution will generate additional E\&G funding. The additional revenues will support growth in the STEM programs. 


\section{PERFORMANCE FUNDING METRICS}

\section{Reported at the USF System Level}




\section{KEY PERFORMANCE INDICATORS}

The Board of Governors has selected the following Key Performance Indicators from its 2025 System Strategic Plan and from accountability metrics identified by the Florida Legislature. The Key Performance Indicators emphasize three primary areas of focus: Academic Quality, Operational Efficiency, and Return on Investment. The indicators address common goals across all universities while also providing flexibility to address institution-specific goals from a list of metrics in the 2025 System Strategic Plan.

The Goals Specific to Research Universities apply only to those universities classified by the Carnegie Foundation for the Advancement of Teaching as being a 'Research University'1, which includes Florida A\&M University (by university request), Florida Atlantic University, Florida International University, Florida State University, University of Central Florida, University of Florida, and the University of South Florida. 


\section{KEY PERFORMANCE INDICATORS}

Metrics Common to All Universities

\begin{tabular}{|c|c|c|c|c|c|c|}
\hline & $\begin{array}{l}\text { FIVE YEAR } \\
\text { TREND }\end{array}$ & $\begin{array}{c}2015 \\
\text { ACTUAL }\end{array}$ & $\begin{array}{l}2016 \\
\text { GOALS }\end{array}$ & $\begin{array}{l}2017 \\
\text { GOALS }\end{array}$ & $\begin{array}{l}2018 \\
\text { GOALS }\end{array}$ & $\begin{array}{c}2019 \\
\text { GOALS }\end{array}$ \\
\hline \multicolumn{7}{|l|}{ Academic Quality } \\
\hline $\begin{array}{l}\text { National Rankings } \\
\text { for University }\end{array}$ & $\mathrm{n} / \mathrm{a}$ & $\begin{array}{c}0 \\
2015\end{array}$ & $\begin{array}{c}0 \\
2016\end{array}$ & $\begin{array}{c}0 \\
2017\end{array}$ & $\begin{array}{c}0 \\
2018\end{array}$ & $\begin{array}{c}0 \\
2019\end{array}$ \\
\hline $\begin{array}{l}\text { SAT Score* } \\
\text { [for } 3 \text { subtests] }\end{array}$ & $\mathrm{n} / \mathrm{a}$ & $\begin{array}{c}1653 \\
\text { Fall } 2014\end{array}$ & $\begin{array}{c}1660 \\
\text { Fall } 2015\end{array}$ & $\mathrm{n} / \mathrm{a}$ & $\mathrm{n} / \mathrm{a}$ & $\mathrm{n} / \mathrm{a}$ \\
\hline High School GPA & $\mathrm{n} / \mathrm{a}$ & $\begin{array}{c}3.78 \\
\text { Fall } 2014\end{array}$ & $\begin{array}{l}3.80 \\
\text { Fall } 2015\end{array}$ & $\begin{array}{l}3.82 \\
\text { Fall } 2016\end{array}$ & $\begin{array}{l}3.83 \\
\text { Fall } 2017\end{array}$ & $\begin{array}{c}3.84 \\
\text { Fall } 2018\end{array}$ \\
\hline $\begin{array}{l}\text { Professional/Licensure Exam } \\
\text { First-time Pass Rates }{ }^{1} \\
\text { Exams Above Benchmarks } \\
\text { Exams Below Benchmarks }\end{array}$ & $\begin{array}{l}\mathrm{n} / \mathrm{a} \\
\mathrm{n} / \mathrm{a}\end{array}$ & \multicolumn{5}{|c|}{ Reported at the USF System Level } \\
\hline \multicolumn{7}{|l|}{ Operational Efficiency } \\
\hline Freshman Retention Rate ${ }^{* *} 1$ & $\mathrm{n} / \mathrm{a}$ & $\begin{array}{c}72 \% \\
2013-14\end{array}$ & $\begin{array}{l}73 \% \\
2014-15\end{array}$ & $\begin{array}{l}74 \% \\
2015-16\end{array}$ & $\begin{array}{l}75 \% \\
2016-17\end{array}$ & $\begin{array}{l}76 \% \\
2017-18\end{array}$ \\
\hline $\begin{array}{l}\text { FTIC Graduation Rates }{ }^{* *} \\
\text { In } 4 \text { years (or less) } \\
\text { In } 6 \text { years (or less) }\end{array}$ & $\begin{array}{l}\mathrm{n} / \mathrm{a} \\
\mathrm{n} / \mathrm{a}\end{array}$ & $\begin{array}{l}\mathrm{n} / \mathrm{a} \\
2010-14 \\
\mathrm{n} / \mathrm{a} \\
2008-14\end{array}$ & $\begin{array}{c}\mathrm{n} / \mathrm{a} \\
2011-15 \\
\mathrm{n} / \mathrm{a} \\
2009-15\end{array}$ & $\begin{array}{c}\mathrm{n} / \mathrm{a} \\
2012-16 \\
\mathrm{n} / \mathrm{a} \\
2010-16\end{array}$ & $\begin{array}{l}40 \% \\
2013-17 \\
\mathrm{n} / \mathrm{a} \\
2011-17\end{array}$ & $\begin{array}{c}41 \% \\
2014-18 \\
\text { n/a } \\
2012-18\end{array}$ \\
\hline $\begin{array}{l}\text { AA Transfer Graduation Rates } \\
\text { In } 2 \text { years (or less) }\end{array}$ & 9 pts & $\begin{array}{l}25 \% \\
2012-14\end{array}$ & $\begin{array}{l}30 \% \\
2013-15\end{array}$ & $\begin{array}{l}31 \% \\
2014-16\end{array}$ & $\begin{array}{l}32 \% \\
2015-17\end{array}$ & $\begin{array}{c}33 \% \\
2016-18\end{array}$ \\
\hline $\begin{array}{l}\text { FTIC Average Time to Degree } \\
\text { (in years) }\end{array}$ & $0.1 \mathrm{pts} \Delta$ & $\begin{array}{c}5.2 \\
2013-14\end{array}$ & $\begin{array}{c}5.2 \\
2014-15\end{array}$ & $\begin{array}{c}5.2 \\
2015-16\end{array}$ & $\begin{array}{c}5.2 \\
2016-17\end{array}$ & $\begin{array}{c}5.2 \\
2017-18\end{array}$ \\
\hline \multicolumn{7}{|l|}{ Return on Investment } \\
\hline $\begin{array}{l}\text { Bachelor's Degrees Awarded } \\
\text { First Majors Only }\end{array}$ & $38 \% \Delta$ & $\begin{array}{c}491 \\
2013-14\end{array}$ & $\begin{array}{c}470 \\
2014-15\end{array}$ & $\begin{array}{c}475 \\
2015-16\end{array}$ & $\begin{array}{c}485 \\
2016-17\end{array}$ & $\begin{array}{c}495 \\
2017-18\end{array}$ \\
\hline $\begin{array}{l}\text { Percent of Bachelor's Degrees } \\
\text { in STEM \& Health }\end{array}$ & 10 pts & $\begin{array}{c}14 \% \\
2013-14\end{array}$ & $\begin{array}{c}15 \% \\
2014-15\end{array}$ & $\begin{array}{c}15 \% \\
2015-16\end{array}$ & $\begin{array}{c}16 \% \\
2016-17\end{array}$ & $\begin{array}{c}16 \% \\
2017-18\end{array}$ \\
\hline Graduate Degrees Awarded & $-7 \% \Delta$ & $\begin{array}{c}55 \\
2013-14\end{array}$ & $\begin{array}{c}49 \\
2014-15\end{array}$ & $\begin{array}{c}50 \\
2015-16\end{array}$ & $\begin{array}{c}50 \\
2016-17\end{array}$ & $\begin{array}{c}50 \\
2017-18\end{array}$ \\
\hline $\begin{array}{l}\text { Percent of Graduate Degrees } \\
\text { in STEM \& Health }\end{array}$ & $\mathrm{n} / \mathrm{a}$ & $\underset{2013-14}{\mathrm{n} / \mathrm{a}}$ & $\begin{array}{c}\mathrm{n} / \mathrm{a} \\
2014-15\end{array}$ & $\begin{array}{c}\mathrm{n} / \mathrm{a} \\
2015-16\end{array}$ & $\begin{array}{c}\mathrm{n} / \mathrm{a} \\
2016-17\end{array}$ & $\begin{array}{c}\mathrm{n} / \mathrm{a} \\
2017-18\end{array}$ \\
\hline
\end{tabular}

\section{Annual Gifts Received}

(\$Millions)

\section{Endowment}

(\$Millions)

Note*: The College Board is revising the SAT test starting March 2016.

${ }^{* *} \mathrm{FTIC}$ retention and graduation rate percentages represent students starting and ending at USFSM.

$183 \%$ of USFSM students of the 2013 cohort were retained within the USF System
Reported at the USF System Level

Reported at the USF System Level 


\section{KEY PERFORMANCE INDICATORS}

\section{Institution Specific Goals}

Each university will provide updates for the metric goals reported in last year's Work Plans. The Board of Governors will consider the shaded 2017 goals for approval. University leadership will need to discuss any proposed changes with Board of Governors staff.

\begin{tabular}{|c|c|c|c|c|c|c|}
\hline & $\begin{array}{l}\text { FIVE YEAR } \\
\text { TREND } \\
2009-10 \text { to } \\
2013-14\end{array}$ & $\begin{array}{c}2015 \\
\text { ACTUAL }\end{array}$ & $\begin{array}{c}2016 \\
\text { GOALS }\end{array}$ & $\begin{array}{c}2017 \\
\text { GOALS }\end{array}$ & $\begin{array}{c}2018 \\
\text { GOALS }\end{array}$ & $\begin{array}{c}2019 \\
\text { GOALS }\end{array}$ \\
\hline $\begin{array}{l}\text { Metric \#1 Number of Adult (age } \\
25+\text { ) Undergraduates Enrolled (in } \\
\text { Fall) }\end{array}$ & $16 \% \Delta$ & $\begin{array}{c}944 \\
2013-14\end{array}$ & $\begin{array}{c}903 \\
2014-15\end{array}$ & $\begin{array}{c}921 \\
2015-16\end{array}$ & $\begin{array}{c}939 \\
2016-17\end{array}$ & $\begin{array}{c}958 \\
2017-18\end{array}$ \\
\hline $\begin{array}{l}\text { Metric \#2 Percent of Course } \\
\text { Sections Offered via Distance and } \\
\text { Blended Learning }\end{array}$ & $11 \mathrm{pts} \Delta$ & $\begin{array}{l}45 \% \\
2013-14\end{array}$ & $\begin{array}{l}42 \% \\
2014-15\end{array}$ & $\begin{array}{l}44 \% \\
2015-16\end{array}$ & $\begin{array}{c}46 \% \\
2016-17\end{array}$ & $\begin{array}{c}48 \% \\
2017-18\end{array}$ \\
\hline $\begin{array}{l}\text { Metric \#3 Seek Carnegie's } \\
\text { Community Engagement } \\
\text { Classification }\end{array}$ & \multicolumn{6}{|c|}{$\begin{array}{l}\text { USF Sarasota-Manatee will submit an application to receive the Elective Community } \\
\text { Engagement Classification in Spring 2019. According to the Carnegie Foundation } \\
\text { timeline, designation will be announced in January } 2020 \text {. }\end{array}$} \\
\hline
\end{tabular}

To further distinguish the university's distinctive mission, the university may choose to provide two additional narrative and metric goals that are based on the university's own strategic plan.

Goal 1. Student Access: Expand access to University education that benefits students and the local, national, and global community

Metric: Lower-Level FTE

$564 \% \Delta$

217
$2013-14$

$\underset{2014-15}{272}$

284
$2015-16$

297

2016-17 2017-18

Goal 2. Student Success: Enhance success of student outcomes, faculty productivity, and community impact.

Metric: 4-year AA Transfer Graduation Rates from State University System
-4 pts $\Delta$

$68 \%$
$2013-14$

\section{$73 \%$
$2014-15$}

$75 \%$
$2015-16$
$77 \%$
$2016-17$
$79 \%$
$2017-18$ 


\section{FISCAL INFORMATION}

University Revenues (in Millions of Dollars)

\begin{tabular}{lcc} 
& $\begin{array}{c}2014-15 \\
\text { Actual }\end{array}$ & $\begin{array}{c}2015-16 \\
\text { Appropriations }\end{array}$ \\
\hline Education \& General - Main Operations & & \\
\hline State Funds & $\$ 14.1$ & Pending \\
\hline Tuition & $\$ 9.0$ & Pending \\
\hline TOTAL MAIN OPERATIONS & $\$ 23.1$ & Pending \\
\hline Education \& General - Health-Science Center / Medical Schools & & \\
\hline State Funds & $\mathrm{n} / \mathrm{a}$ & Pending \\
\hline Tuition & $\mathrm{n} / \mathrm{a}$ & Pending \\
\hline TOTAL HSC & $\mathrm{n} / \mathrm{a}$ & Pending \\
\hline Education \& General - Institute of Food \& Agricultural Sciences (IFAS) & & \\
\hline State Funds & $\mathrm{n} / \mathrm{a}$ & Pending \\
\hline Tuition & $\mathrm{n} / \mathrm{a}$ & Pending \\
\hline TOTAL IFAS & $\mathrm{n} / \mathrm{a}$ & Pending \\
\hline$\quad$ EDUCATION \& GENERAL TOTAL REVENUES & $\$ 23.1$ & Pending \\
\hline
\end{tabular}

Note: State funds include General Revenue funds, Lottery funds, Federal Stimulus funds, and Phosphate Research funds (for Polytechnic) appropriated by the Florida Legislature (as reported in the Annual Accountability Report). Actual tuition includes base tuition and tuition differential fee revenues for resident and non-resident undergraduate and graduate students net of waivers (as reported in the Annual Accountability Report). Actual tuition revenues are not yet available for the 2013-14 year.

\section{OTHER BUDGET ENTITIES}

\section{Auxiliary Enterprises}

Resources associated with auxiliary units that are self-supporting through fees, payments and charges. Examples include housing, food services, bookstores, parking services, health centers.

Revenues $\$ 2.02 \quad$ Pending

\section{Contracts \& Grants}

Resources received from federal, state or private sources for the purposes of conducting research and public service activities.

\begin{tabular}{ccc}
\hline Revenues & $(\$ 0.05)$ & Pending \\
\hline Local Funds & &
\end{tabular}

Resources associated with student activity (supported by the student activity fee), student financial aid, concessions, intercollegiate athletics, technology fee, green fee, and student life \& services fee.
Revenues
$\$ 1.24$
Pending

\section{Faculty Practice Plans}

Revenues/receipts are funds generated from faculty practice plan activities.

Revenues

Reported at USF System Level 


\section{FISCAL INFORMATION (continued)}

\section{Undergraduate Resident Tuition Summary (for 30 credit hours)}

\begin{tabular}{lccccc} 
& $\begin{array}{c}\text { FY 2012-13 } \\
\text { ACTUAL }\end{array}$ & $\begin{array}{c}\text { FY 2013-14 } \\
\text { ACTUAL }\end{array}$ & $\begin{array}{c}\text { FY 2014-15 } \\
\text { ACTUAL }\end{array}$ & $\begin{array}{c}\text { FY 2015-16 } \\
\text { REQUEST }\end{array}$ & $\begin{array}{c}\text { FY 2016-17 } \\
\text { PLANNED }\end{array}$ \\
\hline Base Tuition & $\$ 3,152$ & $\$ 3,152$ & $\$ 3,152$ & $\$ 3,152$ & $\$ 3,152$ \\
\hline Tuition Differential Fee & $\$ 1,054$ & $\$ 1,054$ & $\$ 1,054$ & $\$ 1,054$ & $\$ 1,054$ \\
\hline Percent Increase & $11 \%$ & $1.3 \%$ & $0 \%$ & $0 \%$ & $0 \%$ \\
\hline Required Fees $^{1}$ & $\$ 1,381$ & $\$ 1,381$ & $\$ 1,381$ & $\$ 1,381$ & $\$ 1,381$ \\
\hline TOTAL TUITION AND FEES & $\$ \mathbf{5 , 5 8 7}$ & $\$ \mathbf{5 , 5 8 7}$ & $\mathbf{\$ 5 , 5 8 7}$ & $\$ \mathbf{5 5 , 5 8 7}$ & $\$ \mathbf{5 5 , 5 8 7}$
\end{tabular}

Note $^{1}$ : For more information regarding required fees see list of per credit hour fees and block fees on next page.

\section{Student Debt Summary}

\begin{tabular}{lcccc|c} 
& $\begin{array}{c}2010-11 \\
\text { ACTUAL }\end{array}$ & $\begin{array}{c}2011-12 \\
\text { ACTUAL }\end{array}$ & $\begin{array}{c}2012-13 \\
\text { ACTUAL }\end{array}$ & $\begin{array}{c}2013-14 \\
\text { ACTUAL }\end{array}$ & $\begin{array}{c}2014-15 \\
\text { GOAL }\end{array}$ \\
\hline Percent of Bachelor's Recipients with Debt & $52 \%$ & $52 \%$ & $54 \%$ & $61 \%$ & $60 \%$ \\
\hline $\begin{array}{l}\text { Average Amount of Debt } \\
\text { for Bachelor's who have graduated with debt }\end{array}$ & $\$ 14,448$ & $\$ 17,790$ & $\$ 20,021$ & $\$ 16,351$ & $\$ 16,000$ \\
NSLDS Cohort Year & $\mathbf{2 0 0 9}$ & $\mathbf{2 0 1 0}$ & $\mathbf{2 0 1 1}$ & $\mathbf{2 0 1 2}$ & $\begin{array}{c}\mathbf{2 0 1 3} \\
\text { GOAL }\end{array}$ \\
\hline Student Loan Cohort Default Rate (3rd Year) & $10.10 \%$ & $9.80 \%$ & $7.5 \%$ & $5.4 \%$ est & $5.3 \%$
\end{tabular}

Cost of Attendance (for Full-Time Undergraduate Florida Residents in the Fall and Spring of 2014-15)

\begin{tabular}{ccccccc} 
& $\begin{array}{c}\text { TUITION } \\
\& \text { FEES }\end{array}$ & $\begin{array}{c}\text { BOOKS } \& \\
\text { SUPPLIES }\end{array}$ & $\begin{array}{c}\text { ROOM } \\
\text { BOARD }\end{array}$ & TRANSPORTATION & $\begin{array}{c}\text { OTHER } \\
\text { EXPENSES }\end{array}$ & TOTAL \\
\hline ON-CAMPUS & $\$ 5,588$ & $\$ 1,000$ & $\$ 9,400$ & $\$ 1,500$ & $\$ 2,600$ & $\$ \mathbf{\$ 2 0 , 0 8 8}$ \\
\hline AT HOME & $\$ 5,588$ & $\$ 1,000$ & $\$ 4,700$ & $\$ 1,500$ & $\$ 2,600$ & $\$ 15,388$ \\
\hline
\end{tabular}

*On-campus residence not available.

Estimated Net Cost by Family Income (for Full-Time Undergraduate Florida Residents in the Fall and Spring of 2014-15)

\begin{tabular}{|c|c|c|c|c|c|c|}
\hline \multirow{2}{*}{$\begin{array}{l}\text { FAMILY } \\
\text { INCOME } \\
\text { GROUPS }\end{array}$} & \multicolumn{2}{|c|}{$\begin{array}{l}\text { FULL-TIME RESIDENT } \\
\text { UNDERGRADUATES }\end{array}$} & \multirow{2}{*}{$\begin{array}{c}\text { AVG. NET } \\
\text { COST OF } \\
\text { ATTENDANCE }\end{array}$} & \multirow{2}{*}{$\begin{array}{c}\text { AVG. NET } \\
\text { TUITION } \\
\text { \& FEES } \\
\end{array}$} & \multirow{2}{*}{$\begin{array}{c}\text { AVG. } \\
\text { GIFT AID } \\
\text { AMOUNT }\end{array}$} & \multirow{2}{*}{$\begin{array}{r}\text { AVG. } \\
\text { LOAN } \\
\text { AMOUNT }\end{array}$} \\
\hline & HEADCOUNT & PERCENT & & & & \\
\hline Below $\$ 40,000$ & 237 & $43 \%$ & $\$ 11,710$ & $-\$ 2,225$ & $\$ 7,135$ & $\$ 4,520$ \\
\hline$\$ 40,000-\$ 59,999$ & 74 & $14 \%$ & $\$ 13,758$ & $-\$ 78$ & $\$ 5,028$ & $\$ 2,598$ \\
\hline$\$ 60,000-\$ 79,999$ & 49 & $9 \%$ & $\$ 13,876$ & $\$ 1,319$ & $\$ 3,639$ & $\$ 3,066$ \\
\hline$\$ 80,000-\$ 99,999$ & 29 & $5 \%$ & $\$ 15,238$ & $\$ 2,762$ & $\$ 2,293$ & $\$ 2,541$ \\
\hline$\$ 100,000$ Above & 76 & $14 \%$ & $\$ 15,383$ & $\$ 3,406$ & $\$ 1,621$ & $\$ 2,793$ \\
\hline Missing* & 82 & $15 \%$ & 19475 & $\$ 4,542$ & $\$ 451$ & $\$ 134$ \\
\hline TOTAL & 547 & $100 \%$ & $\$ 13,084^{*}$ & $\$ 444$ & $\$ 4,512$ & $\$ 3,127$ \\
\hline
\end{tabular}

Notes: This data only represents Fall and Spring financial aid data and is accurate as of March 31, 2015. Please note that small changes to Spring 2014 awards are possible before the data is finalized. Family Income Groups are based on the Total Family Income (including untaxed income) as reported on student FAFSA records. Full-time Students is a headcount based on at least 24 credit hours during Fall and Spring terms. Average Gift Aid includes all grants and scholarships from Federal, State, University and other private sources administered by the Financial Aid Office. Student waivers are also included in the Gift Aid amount. Gift Aid does not include the parental contribution towards EFC. Net Cost of Attendance is the actual average of the total Costs of Attendance (which will vary by income group due to the diversity of students living on- \& off- campus) minus the average Gift Aid amount. Net Tuition \& Fees is the actual average of the total costs of tuition and fees (which will vary by income group due to the amount of credit hours students are enrolled) minus the average Gift Aid amount (see page 16 for list of fees that are included). Average Loan Amount includes Federal (Perkins, Stafford, Ford Direct, and PLUS loans) and all private loans. The bottom-line Average represents the average of all full-time undergraduate Florida residents (note*: the total Net Cost of Attendance does not include students with missing family income data). 'Missing' includes students who did not file a FAFSA. 


\section{FISCAL INFORMATION (continued) TUITION DIFFERENTIAL FEE INCREASE REQUEST FOR FALL 2015}

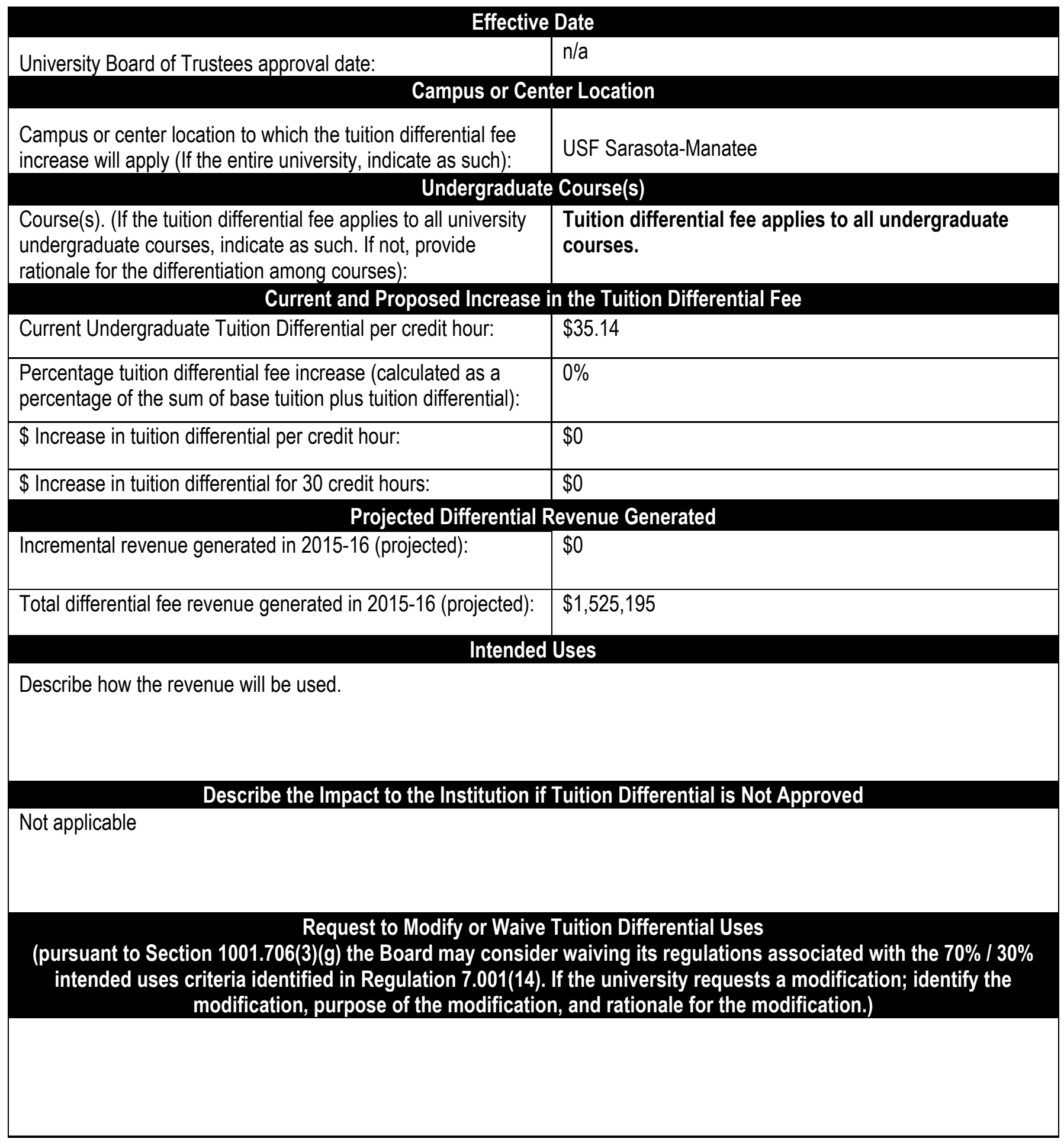




\section{FISCAL INFORMATION (continued) TUITION DIFFERENTIAL SUPPLEMENTAL INFORMATION}

Provide the following information for the 2013-14 academic year.

\begin{tabular}{|c|c|}
\hline $\begin{array}{c}2014-2015-70 \% \text { Initiatives (list the initiatives provided in } \\
\text { the 2013-14 tuition differential request) }\end{array}$ & University Update on Each Initiative \\
\hline Enhance timely college completion rates. & $\begin{array}{l}343 \text { courses funded. } \\
2 \text {-year and } 4 \text {-year graduation rates of AA transfers are } \\
\text { estimated to increase by } 5 \% \text { and } 3 \% \text { respectively over last } \\
\text { year. }\end{array}$ \\
\hline \multicolumn{2}{|c|}{ Additional Detail, where applicable: } \\
\hline $\begin{array}{l}\text { Total Number of Faculty Hired or Retained (funded by tuition } \\
\text { differential): }\end{array}$ & 118 \\
\hline $\begin{array}{l}\text { Total Number of Advisors Hired or Retained (funded by } \\
\text { tuition differential): }\end{array}$ & 0 \\
\hline $\begin{array}{l}\text { Total Number of Course Sections Added or Saved (funded } \\
\text { by tuition differential): }\end{array}$ & 343 \\
\hline $\begin{array}{c}2014-2015-30 \% \text { Initiatives (list the initiatives provided in } \\
\text { the } 2014-15 \text { tuition differential request) }\end{array}$ & University Update on Each Initiative \\
\hline $\begin{array}{l}\text { More students supported with financial aid scholarships and } \\
\text { reduced loan indebtedness. }\end{array}$ & $\begin{array}{l}\text { We were able to maintain our awarding strategy to support } \\
\text { recruitment and retention activities }\end{array}$ \\
\hline $\begin{array}{l}\text { Provide financial support for talented students with limited } \\
\text { income. }\end{array}$ & The average debt of graduates dropped significantly in 13-14. \\
\hline \multicolumn{2}{|c|}{ Additional Information (estimates as of April 1,2015$)$ : } \\
\hline $\begin{array}{l}\text { Unduplicated Count of Students Receiving at least one } \\
\text { Tuition Differential-Funded Award: }\end{array}$ & 365 \\
\hline $\begin{array}{l}\text { \$ Mean (per student receiving an award) of Tuition } \\
\text { Differential-Funded Awards: }\end{array}$ & $\$ 1,077$ \\
\hline $\begin{array}{l}\text { \$ Minimum (per student receiving an award) of Tuition } \\
\text { Differential-Funded Awards: }\end{array}$ & $\$ 38$ \\
\hline $\begin{array}{l}\text { \$ Maximum (per student receiving an award) of Tuition } \\
\text { Differential-Funded Awards: }\end{array}$ & $\$ 2,500$ \\
\hline
\end{tabular}




\section{FISCAL INFORMATION (continued) TUITION DIFFERENTIAL COLLECTIONS, EXPENDITURES, \& AVAILABLE BALANCES - FISCAL YEAR 2014-15 AND 2015-16}

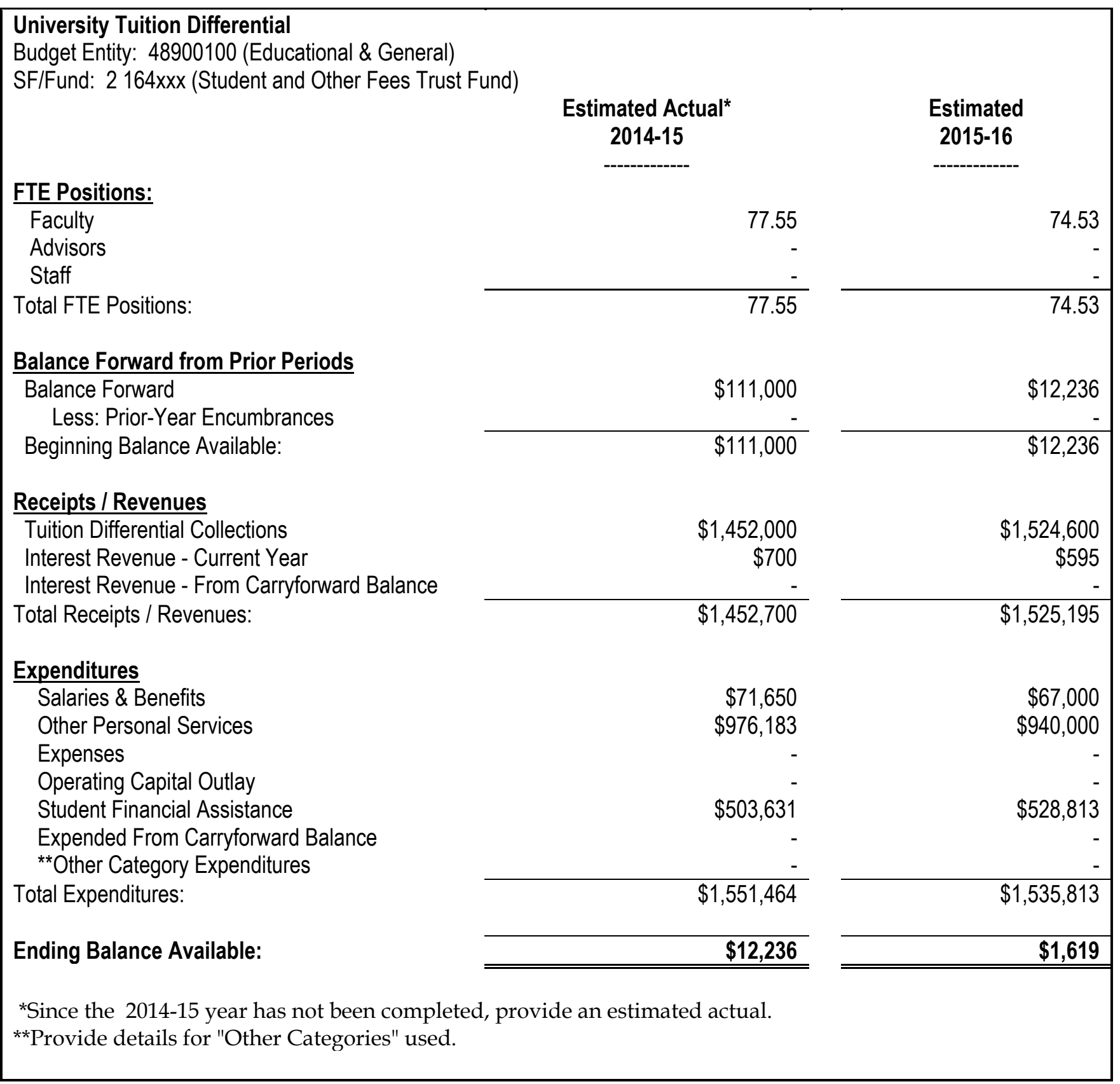




\section{FISCAL INFORMATION (continued) UNIVERSTYY TUITION, FEES AND HOUSING PROJ ECTIONS}

University: University of South Florida Sarasota Manatee

\begin{tabular}{|c|c|c|c|c|c|c|c|}
\hline \multirow[t]{2}{*}{ Undergraduate Students } & \multicolumn{3}{|c|}{ 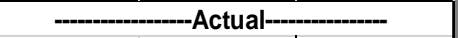 } & \multicolumn{4}{|c|}{ 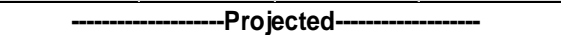 } \\
\hline & 2012-13 & 2013-14 & 2014-15 & 2015-16 & 2016-17 & 2017-18 & 2018-19 \\
\hline \multicolumn{8}{|l|}{ Tuition: } \\
\hline Base Tuition - (0\% inc. for $2014-15$ to $2017-18)$ & $\$ 103.32$ & $\$ 105.07$ & $\$ 105.07$ & $\$ 105.07$ & $\$ 105.07$ & $\$ 105.07$ & $\$ 105.07$ \\
\hline Tuition Differential & $\$ 35.14$ & $\$ 35.14$ & $\$ 35.14$ & $\$ 35.14$ & $\$ 35.14$ & $\$ 35.14$ & $\$ 35.14$ \\
\hline Total Base Tuition \& Differential per Credit Hour & $\$ 138.46$ & $\$ 140.21$ & $\$ 140.21$ & $\$ 140.21$ & $\$ 140.21$ & $\$ 140.21$ & $\$ 140.21$ \\
\hline $\begin{array}{c}\text { \% Change } \\
\end{array}$ & & $1.3 \%$ & $0.0 \%$ & $0.0 \%$ & $0.0 \%$ & $0.0 \%$ & $0.0 \%$ \\
\hline \multicolumn{8}{|l|}{ Fees (per credit hour): } \\
\hline Student Financial Aid ${ }^{1}$ & $\$ 5.16$ & $\$ 5.25$ & $\$ 5.25$ & $\$ 5.25$ & $\$ 5.25$ & $\$ 5.25$ & $\$ 5.25$ \\
\hline Capital Improvement $^{2}$ & $\$ 6.76$ & $\$ 6.76$ & $\$ 6.76$ & $\$ 6.76$ & $\$ 6.76$ & $\$ 6.76$ & $\$ 6.76$ \\
\hline Activity \& Service & $\$ 20.19$ & $\$ 20.19$ & $\$ 20.19$ & $\$ 20.19$ & $\$ 20.19$ & $\$ 20.19$ & $\$ 20.19$ \\
\hline Health & $\$ 4.03$ & $\$ 4.03$ & $\$ 4.03$ & $\$ 4.03$ & $\$ 4.03$ & $\$ 4.03$ & $\$ 4.03$ \\
\hline Athletic & $\$ 4.23$ & $\$ 4.23$ & $\$ 4.23$ & $\$ 4.23$ & $\$ 4.23$ & $\$ 4.23$ & $\$ 4.23$ \\
\hline \multicolumn{8}{|l|}{ Transportation Access } \\
\hline Technology ${ }^{1}$ & $\$ 5.16$ & $\$ 5.25$ & $\$ 5.25$ & $\$ 5.25$ & $\$ 5.25$ & $\$ 5.25$ & $\$ 5.25$ \\
\hline \multicolumn{8}{|l|}{ Green Fee (USF, NCF, UWF only) } \\
\hline \multicolumn{8}{|l|}{ Student Life \& Services Fee (UNF only) } \\
\hline \multicolumn{8}{|l|}{ Marshall Center Fee (USF only) } \\
\hline \multicolumn{8}{|l|}{ Student Affairs Facility Use Fee (FSU only) } \\
\hline Total Fees & $\$ 45.53$ & $\$ 45.71$ & $\$ 45.71$ & $\$ 45.71$ & $\$ 45.71$ & $\$ 45.71$ & $\$ 45.71$ \\
\hline Total Tuition and Fees per Credit Hour & $\$ 183.99$ & $\$ 185.92$ & $\$ 185.92$ & $\$ 185.92$ & $\$ 185.92$ & $\$ 185.92$ & $\$ 185.92$ \\
\hline \begin{tabular}{|c|} 
\% Change \\
\end{tabular} & & $1.0 \%$ & $0.0 \%$ & $0.0 \%$ & $0.0 \%$ & $0.0 \%$ & $0.0 \%$ \\
\hline \multicolumn{8}{|l|}{ Fees (block per term): } \\
\hline \multicolumn{8}{|l|}{ Activity \& Service } \\
\hline \multicolumn{8}{|l|}{ Health } \\
\hline Athletic & $\$ 5.00$ & $\$ 5.00$ & $\$ 5.00$ & $\$ 5.00$ & $\$ 5.00$ & $\$ 5.00$ & $\$ 5.00$ \\
\hline \multicolumn{8}{|l|}{ Transportation Access } \\
\hline \multicolumn{8}{|l|}{ Marshall Center Fee (USF only) } \\
\hline \multicolumn{8}{|l|}{ Student Affairs Facility Use Fee (FSU only) } \\
\hline \multicolumn{8}{|l|}{ List any new fee proposed } \\
\hline Total Block Fees per term & $\$ 5.00$ & $\$ 5.00$ & $\$ 5.00$ & $\$ 5.00$ & $\$ 5.00$ & $\$ 5.00$ & $\$ 5.00$ \\
\hline $\begin{array}{r}\text { \% Change } \\
\end{array}$ & & $0.0 \%$ & $0.0 \%$ & $0.0 \%$ & $0.0 \%$ & $0.0 \%$ & $0.0 \%$ \\
\hline Total Tuition for 30 Credit Hours & $\$ 4,153.80$ & $\$ 4,206.30$ & $\$ 4,206.30$ & $\$ 4,206.30$ & $\$ 4,206.30$ & $\$ 4,206.30$ & $\$ 4,206.30$ \\
\hline Total Fees for 30 Credit Hours & $\$ 1,375.90$ & $\$ 1,381.30$ & $\$ 1,381.30$ & $\$ 1,381.30$ & $\$ 1,381.30$ & $\$ 1,381.30$ & $\$ 1,381.30$ \\
\hline Total Tuition and Fees for 30 Credit Hours & $\$ 5,529.70$ & $\$ 5,587.60$ & $\$ 5,587.60$ & $\$ 5,587.60$ & $\$ 5,587.60$ & $\$ 5,587.60$ & $\$ 5,587.60$ \\
\hline \$Change & & $\$ 57.90$ & $\$ 0.00$ & $\$ 0.00$ & $\$ 0.00$ & $\$ 0.00$ & $\$ 0.00$ \\
\hline$\%$ Change & & $1.0 \%$ & $0.0 \%$ & $0.0 \%$ & $0.0 \%$ & $0.0 \%$ & $0.0 \%$ \\
\hline \multicolumn{8}{|l|}{ Out-of-State Fees } \\
\hline Out-of-State Undergraduate Fee & $\$ 315.00$ & $\$ 346.50$ & $\$ 346.50$ & $\$ 346.50$ & $\$ 346.50$ & $\$ 346.50$ & $\$ 346.50$ \\
\hline Out-of-State Undergraduate Student Financial Aid ${ }^{3}$ & $\$ 15.75$ & $\$ 17.32$ & $\$ 17.32$ & $\$ 17.32$ & $\$ 17.32$ & $\$ 17.32$ & $\$ 17.32$ \\
\hline \begin{tabular}{|c|} 
Total per credit hour \\
\end{tabular} & $\$ 330.75$ & $\$ 363.82$ & $\$ 363.82$ & $\$ 363.82$ & $\$ 363.82$ & $\$ 363.82$ & $\$ 363.82$ \\
\hline \% Change & & $10.0 \%$ & $0.0 \%$ & $0.0 \%$ & $0.0 \%$ & $0.0 \%$ & $0.0 \%$ \\
\hline Total Tuition for $\mathbf{3 0}$ Credit Hours & $\$ 13,603.80$ & $\$ 14,601.30$ & $\$ 14,601.30$ & $\$ 14,601.30$ & $\$ 14,601.30$ & $\$ 14,601.30$ & $\$ 14,601.30$ \\
\hline Total Fees for $\mathbf{3 0}$ Credit Hours & $\$ 1,848.40$ & $\$ 1,900.90$ & $\$ 1,900.90$ & $\$ 1,900.90$ & $\$ 1,900.90$ & $\$ 1,900.90$ & $\$ 1,900.90$ \\
\hline Total Tuition and Fees for 30 Credit Hours & $\$ 15,452.20$ & $\$ 16,502.20$ & $\$ 16,502.20$ & $\$ 16,502.20$ & $\$ 16,502.20$ & $\$ 16,502.20$ & $\$ 16,502.20$ \\
\hline \$Change & & $\$ 1,050.00$ & $\$ 0.00$ & $\$ 0.00$ & $\$ 0.00$ & $\$ 0.00$ & $\$ 0.00$ \\
\hline$\%$ Change & & $6.8 \%$ & $0.0 \%$ & $0.0 \%$ & $0.0 \%$ & $0.0 \%$ & $0.0 \%$ \\
\hline
\end{tabular}




\section{ENROLLMENT PLANNING}

Planned Enrollment Growth by Student Type (for all E\&G students at all campuses)

\begin{tabular}{|c|c|c|c|c|c|c|c|c|c|}
\hline \multirow{2}{*}{ UNDERGRADUATE } & \multirow[t]{2}{*}{$\begin{array}{c}5 \text { YEAR } \\
\text { TREND } \\
\text { (2009-14) }\end{array}$} & \multicolumn{2}{|c|}{$\begin{array}{c}\text { Fall } 2014 \\
\text { ACTUAL } \\
\text { HEADCOUNT }\end{array}$} & \multicolumn{2}{|c|}{$\begin{array}{c}\text { Fall } 2015 \\
\text { PLANNED } \\
\text { HEADCOUNT }\end{array}$} & \multicolumn{2}{|c|}{$\begin{array}{c}\text { Fall } 2016 \\
\text { PLANNED } \\
\text { HEADCOUNT }\end{array}$} & \multicolumn{2}{|c|}{$\begin{array}{c}\text { Fall } 2017 \\
\text { PLANNED } \\
\text { HEADCOUNT }\end{array}$} \\
\hline & & & & & & & & & \\
\hline FTIC (Regular Admit)* & $\mathrm{n} / \mathrm{a}$ & 195 & $11 \%$ & 264 & $14 \%$ & 355 & $18 \%$ & 425 & $20 \%$ \\
\hline FTIC (Profile Admit) & $\mathrm{n} / \mathrm{a}$ & 1 & $0 \%$ & 1 & $0 \%$ & 5 & $0 \%$ & 5 & $0 \%$ \\
\hline AA Transfers from FCS & $-.4 \% \Delta$ & 789 & $56 \%$ & 817 & $54 \%$ & 845 & $52 \%$ & 875 & $50 \%$ \\
\hline Other Transfers & $14 \% \Delta$ & 712 & $33 \%$ & 737 & $32 \%$ & 763 & $30 \%$ & 789 & $30 \%$ \\
\hline Subtotal & $20 \% \Delta$ & 1,697 & $100 \%$ & 1,819 & $100 \%$ & 1,968 & $100 \%$ & 2,094 & $100 \%$ \\
\hline \multicolumn{10}{|l|}{ GRADUATE* $^{*}$} \\
\hline Master's & $-41 \% \Delta$ & 120 & $100 \%$ & 125 & $100 \%$ & 129 & $100 \%$ & 134 & $100 \%$ \\
\hline Research Doctoral & n/a & 0 & $0 \%$ & 0 & $0 \%$ & 0 & $0 \%$ & 0 & $0 \%$ \\
\hline Professional Doctoral & $\mathrm{n} / \mathrm{a}$ & 0 & $0 \%$ & 0 & $0 \%$ & 0 & $0 \%$ & 0 & $0 \%$ \\
\hline Subtotal & $-41 \% \Delta$ & 120 & $100 \%$ & 125 & $100 \%$ & 129 & $100 \%$ & 134 & $100 \%$ \\
\hline \multicolumn{10}{|l|}{ UNCLASSIFIED } \\
\hline H.S. Dual Enrolled & $\mathrm{n} / \mathrm{a}$ & 0 & $0 \%$ & 0 & $0 \%$ & 0 & $0 \%$ & 0 & $0 \%$ \\
\hline Other & $-40 \% \Delta$ & 100 & $100 \%$ & 104 & $100 \%$ & 107 & $100 \%$ & 111 & $100 \%$ \\
\hline Subtotal & $-40 \% \Delta$ & 100 & $100 \%$ & 104 & $100 \%$ & 107 & $100 \%$ & 111 & $100 \%$ \\
\hline TOTAL & $7 \% \Delta$ & 1,917 & & 2,047 & & 2,204 & & 2,339 & \\
\hline
\end{tabular}

Note*: Includes Medical students.

Planned Enrollment Growth by Method of Instruction (for all E\&G students at all campuses)

\begin{tabular}{|c|c|c|c|c|c|c|c|c|c|}
\hline & \multirow{2}{*}{$\begin{array}{c}3 \text { YEAR } \\
\text { TREND } \\
\text { (2010-11 to } \\
2013-14)\end{array}$} & \multicolumn{2}{|c|}{ 2013-14 } & \multicolumn{2}{|c|}{ 2014-15 } & \multicolumn{2}{|c|}{$2015-16$} & \multicolumn{2}{|c|}{ 2016-17 } \\
\hline & & $\begin{array}{c}\text { ACTUAL } \\
\text { FTE }\end{array}$ & $\begin{array}{c}\% \text { of } \\
\text { TOTAL }\end{array}$ & $\begin{array}{l}\text { PLANNED } \\
\text { FTE }\end{array}$ & $\begin{array}{c}\% \text { of } \\
\text { TOTAL }\end{array}$ & $\begin{array}{l}\text { PLANNED } \\
\text { FTE }\end{array}$ & $\begin{array}{c}\% \text { of } \\
\text { TOTAL }\end{array}$ & $\begin{array}{c}\text { PLANNED } \\
\text { FTE }\end{array}$ & $\begin{array}{c}\% \text { of } \\
\text { TOTAL }\end{array}$ \\
\hline \multicolumn{10}{|l|}{ UNDERGRADUATE } \\
\hline DISTANCE (>80\%) & $13 \% \Delta$ & 529 & $50 \%$ & 545 & $50 \%$ & 580 & $50 \%$ & 606 & $50 \%$ \\
\hline HYBRID (50\%-79\%) & $68 \% \Delta$ & 45 & $4 \%$ & 24 & $2 \%$ & 46 & $4 \%$ & 48 & $4 \%$ \\
\hline TRADITIONAL (<50\%) & $-15 \% \Delta$ & 489 & $46 \%$ & 536 & $48 \%$ & 533 & $46 \%$ & 557 & $46 \%$ \\
\hline TOTAL & $-1 \% \Delta$ & 1,064 & $100 \%$ & 1105 & $100 \%$ & 1,159 & $100 \%$ & 1,211 & $100 \%$ \\
\hline \multicolumn{10}{|l|}{ GRADUATE } \\
\hline DISTANCE (80\%) & $-6 \% \Delta$ & 22 & $23 \%$ & 29 & $32 \%$ & 32 & $33 \%$ & 33 & $33 \%$ \\
\hline HYBRID (50\%-79\%) & $72 \% \Delta$ & 4 & $4 \%$ & 4 & $4 \%$ & 4 & $4 \%$ & 4 & $4 \%$ \\
\hline TRADITIONAL (<50\%) & $-29 \% \Delta$ & 71 & $73 \%$ & 58 & $64 \%$ & 60 & $63 \%$ & 64 & $63 \%$ \\
\hline TOTAL & $-23 \% \Delta$ & 98 & $100 \%$ & 91 & $100 \%$ & 96 & $100 \%$ & 101 & $100 \%$ \\
\hline
\end{tabular}

Note: Full-time Equivalent (FTE) student is a measure of instructional effort (and student activity) that is based on the number of credit hours that students enroll. FTE is based on the Florida definition, which divides undergraduate credit hours by 40 and graduate credit hours by 32 . Distance Learning is a course in which at least 80 percent of the direct instruction of the course is delivered using some form of technology when the student and instructor are separated by time or space, or both (per 1009.24(17), F.S.). Hybrid is a course where $50 \%$ to $79 \%$ of the instruction is delivered using some form of technology, when the student and instructor are separated by time or space, or both (per SUDS data element 2052). Traditional (and Technology Enhanced) refers to primarily face to face instruction utilizing some form of technology for delivery of supplemental course materials for no more than $49 \%$ of instruction (per SUDS data element 2052). 


\section{ENROLLMENT PLANNING (continued)}

\section{Planned Enrollment Plan by Residency and Student Level (Florida FTE)}

\begin{tabular}{|c|c|c|c|c|c|c|c|c|c|}
\hline & $\begin{array}{c}\text { Estimated } \\
\text { Actual } \\
\text { 2014-15 } \\
\end{array}$ & $\begin{array}{l}\text { Funded } \\
2015-16 \\
\end{array}$ & $\begin{array}{l}\text { Planned } \\
2015-16 \\
\end{array}$ & $\begin{array}{l}\text { Planned } \\
2016-17 \\
\end{array}$ & $\begin{array}{c}\text { Planned } \\
2017-18\end{array}$ & $\begin{array}{c}\text { Planned } \\
2018-19 \\
\end{array}$ & $\begin{array}{l}\text { Planned } \\
2019-20 \\
\end{array}$ & $\begin{array}{l}\text { Planned } \\
2020-21 \\
\end{array}$ & $\begin{array}{c}\text { Planned } \\
\text { Annual } \\
\text { Growth } \\
\text { Rate }^{*}\end{array}$ \\
\hline \multicolumn{10}{|c|}{ STATE FUNDABLE } \\
\hline \multicolumn{10}{|c|}{ Florida Resident } \\
\hline LOWER & 254 & $\mathrm{n} / \mathrm{a}$ & 266 & 278 & 290 & 303 & 317 & 331 & $4.5 \%$ \\
\hline UPPER & 804 & $\mathrm{n} / \mathrm{a}$ & 842 & 880 & 919 & 960 & 1,004 & 1,049 & $4.5 \%$ \\
\hline GRAD I & 86 & $\mathrm{n} / \mathrm{a}$ & 90 & 94 & 98 & 103 & 107 & 112 & $4.5 \%$ \\
\hline GRAD II & $\mathrm{n} / \mathrm{a}$ & $\mathrm{n} / \mathrm{a}$ & $\mathrm{n} / \mathrm{a}$ & $\mathrm{n} / \mathrm{a}$ & $\mathrm{n} / \mathrm{a}$ & $\mathrm{n} / \mathrm{a}$ & $\mathrm{n} / \mathrm{a}$ & $\mathrm{n} / \mathrm{a}$ & n/a \\
\hline TOTAL & 1144 & $\mathrm{n} / \mathrm{a}$ & 1,198 & 1,252 & 1,307 & 1,366 & 1,428 & 1,492 & $4.5 \%$ \\
\hline \multicolumn{10}{|c|}{ Non-Resident } \\
\hline LOWER & 17 & $\mathrm{n} / \mathrm{a}$ & 18 & 19 & 20 & 21 & 22 & 23 & $5 \%$ \\
\hline UPPER & 30 & $\mathrm{n} / \mathrm{a}$ & 33 & 35 & 36 & 38 & 40 & 42 & $4.9 \%$ \\
\hline GRAD I & 6 & $\mathrm{n} / \mathrm{a}$ & 6 & 6 & 6 & 7 & 7 & 7 & $3.3 \%$ \\
\hline GRAD II & $\mathrm{n} / \mathrm{a}$ & $\mathrm{n} / \mathrm{a}$ & $\mathrm{n} / \mathrm{a}$ & $\mathrm{n} / \mathrm{a}$ & $\mathrm{n} / \mathrm{a}$ & $\mathrm{n} / \mathrm{a}$ & $\mathrm{n} / \mathrm{a}$ & $\mathrm{n} / \mathrm{a}$ & n/a \\
\hline TOTAL & 53 & $\mathrm{n} / \mathrm{a}$ & 57 & 60 & 62 & 66 & 69 & 72 & $4.8 \%$ \\
\hline \multicolumn{10}{|l|}{ TOTAL } \\
\hline LOWER & 271 & 0 & 284 & 297 & 310 & 324 & 338 & 354 & $4.5 \%$ \\
\hline UPPER & 834 & 798 & 875 & 914 & 955 & 999 & 1,044 & 1,090 & $4.5 \%$ \\
\hline GRAD I & 92 & 182 & 96 & 100 & 104 & 109 & 114 & 119 & $4.4 \%$ \\
\hline GRAD ॥ & $\mathrm{n} / \mathrm{a}$ & $\mathrm{n} / \mathrm{a}$ & $\mathrm{n} / \mathrm{a}$ & $\mathrm{n} / \mathrm{a}$ & $\mathrm{n} / \mathrm{a}$ & $\mathrm{n} / \mathrm{a}$ & $\mathrm{n} / \mathrm{a}$ & $\mathrm{n} / \mathrm{a}$ & n/a \\
\hline TOTAL & 1,197 & 980 & 1,255 & 1,312 & 1,369 & 1,432 & 1,497 & 1,564 & $4.5 \%$ \\
\hline \multicolumn{10}{|c|}{ NOT STATE FUNDABLE } \\
\hline LOWER & 3 & $\mathrm{n} / \mathrm{a}$ & 3 & 3 & 3 & 3 & 3 & 3 & $0 \%$ \\
\hline UPPER & 30 & $\mathrm{n} / \mathrm{a}$ & 30 & 31 & 33 & 34 & 36 & 37 & $4.3 \%$ \\
\hline GRAD I & 4 & $\mathrm{n} / \mathrm{a}$ & 4 & 4 & 4 & 4 & 4 & 5 & $5.0 \%$ \\
\hline GRAD ॥ & $\mathrm{n} / \mathrm{a}$ & $\mathrm{n} / \mathrm{a}$ & $\mathrm{n} / \mathrm{a}$ & $\mathrm{n} / \mathrm{a}$ & $\mathrm{n} / \mathrm{a}$ & $\mathrm{n} / \mathrm{a}$ & $\mathrm{n} / \mathrm{a}$ & $\mathrm{n} / \mathrm{a}$ & $\mathrm{n} / \mathrm{a}$ \\
\hline TOTAL & 37 & n/a & 37 & 38 & 40 & 41 & 43 & 45 & $4.0 \%$ \\
\hline
\end{tabular}

Note: Full-time Equivalent (FTE) student is a measure of instructional effort (and student activity) that is based on the number of credit hours that students enroll. FTE is based on the Florida definition, which divides undergraduate credit hours by 40 and graduate credit hours by 32 . Note*:The average annual growth rate is based on the annual growth rate from 2015-16 to 202021. 


\section{ACADEMC PROGRAM COORDINATION}

New Programs For Consideration by University in AY 2015-16

The S.U.S. Council of Academic Vice Presidents (CAVP) Academic Program Coordination Work Group will review these programs as part of their on-going coordination efforts. The programs listed below are based on the 2014-15 Work Plan list for programs under consideration for 2015-16.

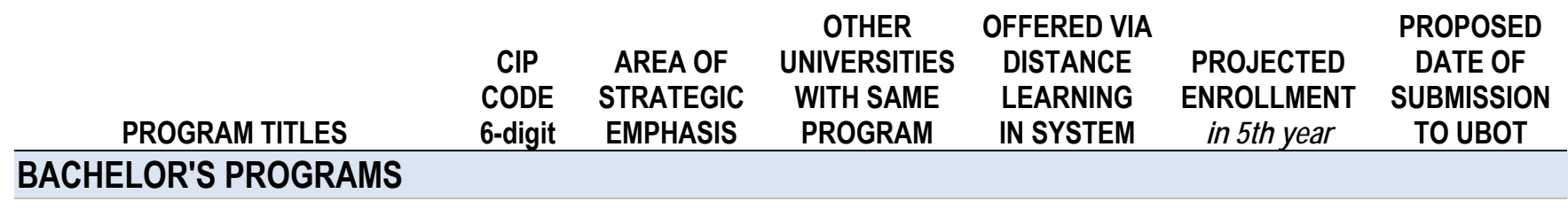

\section{MASTER'S, SPECIALIST AND OTHER ADVANCED MASTER'S PROGRAMS}

\section{DOCTORAL PROGRAMS}

New Programs For Consideration by University in 2016-18

These programs will be used in the 2016 Work Plan list for programs under consideration for 2016-17.

\begin{tabular}{|c|c|c|c|c|c|c|}
\hline PROGRAM TITLES & $\begin{array}{l}\text { CIP } \\
\text { CODE } \\
\text { 6-digit }\end{array}$ & $\begin{array}{l}\text { AREA OF } \\
\text { STRATEGIC } \\
\text { EMPHASIS }\end{array}$ & $\begin{array}{c}\text { OTHER } \\
\text { UNIVERSITIES } \\
\text { WITH SAME } \\
\text { PROGRAM }\end{array}$ & $\begin{array}{l}\text { OFFERED VIA } \\
\text { DISTANCE } \\
\text { LEARNING } \\
\text { IN SYSTEM }\end{array}$ & $\begin{array}{l}\text { PROJECTED } \\
\text { ENROLLMENT } \\
\text { in } 5 \text { th year }\end{array}$ & $\begin{array}{l}\text { PROPOSED } \\
\text { DATE OF } \\
\text { SUBMISSION } \\
\text { TO UBOT }\end{array}$ \\
\hline CHELOR'S PROGRAMS & & & & & & \\
\hline
\end{tabular}

\section{MASTER'S, SPECIALIST AND OTHER ADVANCED MASTER'S PROGRAMS}




\section{DEFINITIONS}

\section{Performance Based Funding}

\author{
Percent of Bachelor's \\ Graduates Employed Full- \\ time or Continuing their \\ Education in the U.S. One \\ Year After Graduation
}

This metric is based on the percentage of a graduating class of bachelor's degree recipients who are employed full-time or continuing their education somewhere in the United States. Students who do not have valid social security numbers and are not found enrolled are excluded. Note: This data now non-Florida employment data.

Sources: State University Database System (SUDS), Florida Education \& Training Placement Information Program (FETPIP) analysis of Wage Record Interchange System (WRIS2) and Federal Employment Data Exchange (FEDES), and National Student Clearinghouse (NSC).

\section{Median Wages of Bachelor's Graduates Employed Full-time in Florida One Year After Graduation}

\section{Average Cost} per Bachelor's Degree Instructional costs to the university
This metric is based on annualized Unemployment Insurance (UI) wage data from the fourth fiscal quarter after graduation for bachelor's recipients. UI wage data does not include individuals who are self-employed, employed out of state, employed by the military or federal government, those without a valid social security number, or making less than minimum wage. Sources: State University Database System (SUDS), Florida Education \& Training Placement Information Program (FETPIP), National Student Clearinghouse.
For each of the last four years of data, the annual total undergraduate instructional expenditures were divided by the total fundable student credit hours to create a cost per credit hour for each year. This cost per credit hour was then multiplied by 30 credit hours to derive an average annual cost. The average annual cost for each of the four years was summed to provide an average cost per degree for a baccalaureate degree that requires 120 credit hours. Sources: State University Database System (SUDS), Expenditure Analysis: Report IV.

\section{Six Year FTIC Graduation Rate}

\section{Academic}

Progress Rate

2nd Year Retention

with GPA Above 2.0

University Access Rate

Percent of Undergraduates with a Pell-grant

\section{Bachelor's}

Degrees Awarded within Programs of Strategic Emphasis (includes STEM)

\section{Graduate}

Degrees Awarded within Programs of Strategic Emphasis (includes STEM)
This metric is based on the percentage of first-ime-in-college (FTIC) students who started in the Fall (or summer continuing to Fall) term and had graduated from the same institution within six years. Students of degree programs longer than four years (eg, PharmD) are included in the cohorts. Students who are active duty military are not included in the data.

Source: State University Database System (SUDS).

This metric is based on the percentage of first-time-in-college (FTIC) students who started in the Fall (or summer continuing to Fall) term and were enrolled full-time in their first semester and were still enrolled in the same institution during the Fall term following their first year with had a grade point average (GPA) of at least 2.0 at the end of their first year (Fall, Spring, Summer).

Source: State University Database System (SUDS).

This metric is based the number of undergraduates, enrolled during the fall term, who received a Pell-grant during the fall term. Unclassified students, who are not eligible for Pell-grants, were excluded from this metric. Source: State University Database System (SUDS).

This metric is based on the number of baccalaureate degrees awarded within the programs designated by the Board of Governors as 'Programs of Strategic Emphasis'. A student who has multiple majors in the subset of targeted Classification of Instruction Program codes will be counted twice (i.e., double-majors are included).

Source: State University Database System (SUDS).

This metric is based on the number of graduate degrees awarded within the programs designated by the Board of Governors as 'Programs of Strategic Emphasis'. A student who has multiple majors in the subset of targeted Classification of Instruction Program codes will be counted twice (i.e., double-majors are included).

Source: State University Database System (SUDS). 


\author{
Freshmen in Top $10 \%$ of \\ High School Class \\ Applies to: NCF
}

\section{BOG Choice Metrics}

Percent of all degree-seeking, first-time, first-year (freshman) students who had high school class rank within the top $10 \%$ of their graduating high school class.

Source: New College of Florida.

\section{Percent of Bachelor's Degrees Without Excess Hours}

This metric is based on the percentage of baccalaureate degrees awarded within $110 \%$ of the credit hours required for a degree based on the Board of Governors Academic Program Inventory.

Note: It is important to note that the statutory provisions of the "Excess Hour Surcharge" $(1009.286, \mathrm{FS})$ have been modified several times by the Florida Legislature, resulting in a phased-in approach that has created three different cohorts of students with different requirements. The performance funding metric data is based on the latest statutory requirements that mandates $110 \%$ of required hours as the threshold. In accordance with statute, this metric excludes the following types of student credits (ie, accelerated mechanisms, remedial coursework, non-native credit hours that are not used toward the degree, non-native credit hours from failed, incomplete, withdrawn, or repeated courses, credit hours from internship programs, credit hours up to 10 foreign language credit hours, and credit hours earned in military science courses that are part of the Reserve Officers' Training Corps (ROTC) program).

Source: State University Database System (SUDS).

This metric is based on the number of awards that faculty have earned in the arts, humanities, science, engineering and health fields as reported in the annual 'Top American Research Universities' report. Twenty-three of the most prominent awards are considered, including: Getty Scholars in Residence, Guggenheim Fellows, Howard Hughes Medical Institute Investigators, MacArthur Foundation Fellows, National Endowment for the Humanities (NEH) Fellows, National Medal of Science and National Medal of Technology, Robert Wood Johnson Policy Fellows, Sloan Research Fellows, Woodrow Wilson Fellows, to name a few awards. Source: Center for Measuring University Performance, Annual Report of the Top American Research Universities (TARU).

This metric is based on the number of Top 50 university rankings that NCF earned from the following list of publications: Princeton Review, Fiske Guide, QS World University Ranking, Times Higher Education World University Ranking, Academic Ranking of World University, US News and World Report National University, US News and World Report National Public University, US News and World Report Liberal Arts Colleges, Forbes, Kiplinger, Washington Monthly Liberal Arts Colleges, Washington Monthly National University, and Center for Measuring University Performance. Source: Board of Governors staff review.

\section{BOT Choice Metrics}

\section{Percent of R\&D Expenditures Funded from External Sources FAMU}

Bachelor's Degrees
Awarded to Minorities
FAU, FGCU, FIU

National Rank Higher than Predicted by the Financial Resources Ranking Based on U.S. and World News FSU
This metric reports the amount of research expenditures that was funded from federal, private industry and other (non-state and non-institutional) sources.

Source: National Science Foundation annual survey of Higher Education Research and Development (HERD).

This metric is the number, or percentage, of baccalaureate degrees granted in an academic year to Non-Hispanic Black and Hispanic students. This metric does not include students classified as Non-Resident Alien or students with a missing race code. Source: State University Database System (SUDS).
This metric is based on the difference between the Financial Resources rank and the overall University rank. U.S. News measures financial resources by using a two-year average spending per student on instruction, research, student services and related educational expenditures - spending on sports, dorms and hospitals doesn't count. Source: US News and World Report's annual National University rankings. 


\section{Percent of Undergraduate Seniors Participating in a Research Course NCF}

Number of Bachelor
Degrees Awarded Annually
UCF

This metric is based on the percentage of undergraduate seniors who participate in a research course during their senior year.

Source: New College of Florida.

This metric is the number of baccalaureate degrees granted in an academic year. Students who earned two distinct degrees in the same academic year were counted twice; students who completed multiple majors or tracks were only counted once.

Source: State University Database System (SUDS).

This metric is the total expenditures (includes non-science \& engineering fields) for research \& development activities within a given fiscal year.

Source: National Science Foundation annual survey of Higher Education Research and Development (HERD).

Expenditures

UF

Percent of Course Sections
Offered via Distance and
Blended Learning
UNF

Number of Postdoctoral
Appointees
USF

This metric is based on the percentage of course sections classified as having at least $50 \%$ of the instruction delivered using some form of technology, when the student and instructor are separated by time or space, or both. Source: State University Database System (SUDS).

This metric is based on the number of post-doctoral appointees at the beginning of the academic year. A postdoctoral researcher has recently earned a doctoral (or foreign equivalent) degree and has a temporary paid appointment to focus on specialized research/scholarship under the supervision of a senior scholar.

Source: National Science Foundation/National Institutes of Health annual Survey of Graduate Students and Postdoctorates in Science and Engineering (GSS).

\section{Percentage of Adult Undergraduates Enrolled UWF}

This metric is based on the percentage of undergraduates (enrolled during the fall term) who are at least 25 years old at the time of enrollment. This includes undergraduates who are not degree-seeking, or unclassified.

Source: State University Database System (SUDS).

\section{Preeminent Research University Funding Metrics}

Average GPA and SAT Score

An average weighted grade point average of 4.0 or higher and an average SAT score of 1800 or higher for fall semester incoming freshmen, as reported annually in the admissions data that universities submit to the Board of Governors. This data includes registered FTIC (student type='B','E') with an admission action of admitted or provisionally admitted ('A','P','X').

Public University National
Ranking

Freshman Retention Rate (Full-time, FTIC)
A top-50 ranking on at least two well-known and highly respected national public university rankings, reflecting national preeminence, using most recent rankings, includes: Princeton Review, Fiske Guide, QS World University Ranking, Times Higher Education World University Ranking, Academic Ranking of World University, US News and World Report National University, US News and World Report National Public University, US News and World Report Liberal Arts Colleges, Forbes, Kiplinger, Washington Monthly Liberal Arts Colleges, Washington Monthly National University, and Center for Measuring University Performance.

Freshman Retention Rate (Full-time, FTIC) as reported annually to the Integrated Postsecondary Education Data System (IPEDS). The retention rates that are reported in the Board's annual Accountability report are preliminary because they are based on student enrollment in their second fall term as reported by the 28th calendar day following the first day of class. When the Board of Governors reports final retention rates to IPEDS in the Spring (usually the first week of April), that data is based on the student enrollment data as reported after the Fall semester has been completed. The preliminary and final retention rates are nearly identical when rounded to the nearest whole number. 
Cohorts are based on undergraduate students who enter the institution in the Fall term (or Summer term and continue into the Fall term). Percent Graduated is based on federal rate and does not include students who originally enroll as part-time students, or who transfer into the

6-year Graduation Rate (Full-time, FTIC) institution. This metric complies with the requirements of the federal Student Right to Know Act that requires institutions to report the completion status at $150 \%$ of normal time (or six years). For more information about how this data is calculated, see: http://www.flbog.edu/about/budget/docs/performance_funding/PBF_GRADUATION_and_RETENTION_ Methodology_FINAL.pdf.

\section{National Academy} Memberships
Total Annual Research Expenditures (\$M) (Science \& Engineering only)

Total Annual Research Expenditures in Diversified Non-Medical Sciences (\$M) (Science \& Engineering only)

National Ranking in S.T.E.M. Research Expenditures

National Academy Memberships held by faculty as reported by the Center for Measuring University Performance in the Top American Research Universities (TARU) annual report.

Total Science \& Engineering Research Expenditures, including federal research expenditures, of $\$ 200$ million or more, as reported annually by the National Science Foundation (NSF).

Total S\&E research expenditures in non-medical sciences as reported by the NSF. This removes medical sciences funds ( $9 F \& 12 F$ in HERD survey) from the total $S \& E$ amount.

The NSF identifies 8 broad disciplines within Science \& Engineering (Computer Science, Engineering, Environmental Science, Life Science, Mathematical Sciences, Physical Sciences, Psychology, Social Sciences). The rankings by discipline are determined by BOG staff using the NSF WebCaspar database.

Total patents awarded by the United States Patent and Trademark Office (USPTO) for the most recent 3-year period. Due to a year-lag in published reports, Board of Governors staff query the USPTO database with a query that only counts utility patents:"(AN/"University Name" AND ISD/yyyymmdd->yyyymmdd AND APT/1)".

Doctoral degrees awarded annually, as reported annually in the Board of Governors Accountability Report. Note: per legislative workpapers, this metric does not include Professional degrees.

Annually

The number of Postdoctoral Appointees awarded annually, as reported in the TARU annual

Number of Post-Doctoral Appointees report. This data is based on National Science Foundation/National Institutes of Health annual Survey of Graduate Students and Postdoctorates in Science and Engineering (GSS).

This data comes from the National Association of College and University Business Officers Endowment Size (\$M) (NACUBO) and Commonfund Institute's annual report of Market Value of Endowment Assets which, due to timing, may release the next fiscal year's data after the Board of Governors Accountability report is published. 


\section{Goals Common to All Universities \\ Academic Quality}

Avg. SAT Score (for 3 subtests)

An average weighted grade point average of 4.0 or higher and an average SAT score of 1800 or higher for fall semester incoming freshmen, as reported annually in the admissions data that universities submit to the Board of Governors. This data includes registered FTIC (student type='B','E') with an admission action of admitted or provisionally admitted ('A','P','X').

Avg. HS GPA

Professional/Licensure Exam First-time Pass Rates

Operational Efficiency

Freshman Retention Rate

FTIC Graduation Rates In 4 years (or less)

In 6 years (or less)

\section{AA Transfer Graduation Rates} In 2 years (or less)

\section{Average Time to Degree (for FTIC)}

\section{Return on Investment}

Bachelor's Degrees Awarded

\section{Percent of Bachelor's Degrees in STEM}

Graduate Degrees Awarded

Percent of Graduate Degrees in STEM

Annual Gifts Received (\$M)

Endowment $(\$ M)$
The average HS GPA for Admitted \& Registered FTIC and early admit $(B, E)$ students. Max score is 5.0 .

The number of exams with first-time pass rates above and below the national or state average, as reported in the annual Accountability report, including: Nursing, Law, Medicine (3 subtests), Veterinary, Pharmacy, Dental (2 subtests), Physical Therapy, and Occupational Therapy.

The percentage of a full-time, first-time-in-college (FTIC) undergraduate cohort (entering in fall term or summer continuing to fall) that is still enrolled or has graduated from the same institution in the following fall term as reported in the annual Accountability report (table 4B) - see link.

As reported in the annual Accountability report (table 4D), First-time-in-college (FTIC) cohort is defined as undergraduates entering in fall term (or summer continuing to fall) with fewer than 12 hours earned since high school graduation. The rate is the percentage of the initial cohort that has either graduated from or is still enrolled in the same institution by the fourth or sixth academic year. Both full-time and part-time students are used in the calculation. The initial cohort is revised to remove students, who have allowable exclusions as defined by IPEDS, from the cohort.

As reported in the annual Accountability report (table 4E), AA Transfer cohort is defined as undergraduates entering in the fall term (or summer continuing to fall) and having earned an $A A$ degree from an institution in the Florida College System. The rate is the percentage of the initial cohort that has either graduated from or is still enrolled in the same institution by the second or fourth academic year. Both full-time and part-time students are used in the calculation. The initial cohort is revised to remove students, who have allowable exclusions as defined by IPEDS, from the cohort.

This metric is the number of years between the start date (using date of most recent admission) and the end date (using the last month in the term degree was granted) for a graduating class of first-time, single-major baccalaureates in 120 credit hour programs within a (Summer, Fall, Spring) year.

This is a count of baccalaureate degrees awarded as reported in the annual Accountability Report (table 4G).

The percentage of baccalaureate degrees that are classified as STEM by the Board of Governors in the SUS program inventory as reported in the annual Accountability Report (table $4 \mathrm{H})$.

This is a count of graduate degrees awarded as reported in the Accountability Report (table 5B).

The percentage of baccalaureate degrees that are classified as STEM by the Board of Governors in the SUS program inventory as reported in the annual Accountability Report (table $5 C)$.

As reported in the Council for Aid to Education's Voluntary Support of Education (VSE) survey in the section entitled "Gift Income Summary," this is the sum of the present value of all gifts (including outright and deferred gifts) received for any purpose and from all sources during the fiscal year, excluding pledges and bequests. (There's a deferred gift calculator at www.cae.org/vse.) The present value of non-cash gifts is defined as the tax deduction to the donor as allowed by the IRS.

Endowment value at the end of the fiscal year, as reported in the annual NACUBO Endowment Study (changed to the NACUBO-Common Fund Study of Endowments in 2009). 


\section{Goals Specific to Research Universities}

Academic Quality

Awards include: American Council of Learned Societies (ACLS) Fellows, Beckman Young

Investigators, Burroughs Wellcome Fund Career Awards, Cottrell Scholars, Fulbright American

Scholars, Getty Scholars in Residence, Guggenheim Fellows, Howard Hughes Medical Institute Investigators, Lasker Medical Research Awards, MacArthur Foundation Fellows, Andrew W. Mellon Foundation Distinguished Achievement Awards, National Endowment for

Faculty Awards the Humanities (NEH) Fellows, National Humanities Center Fellows, National Institutes of Health (NIH) MERIT, National Medal of Science and National Medal of Technology, NSF CAREER awards (excluding those who are also PECASE winners), Newberry Library Longterm Fellows, Pew Scholars in Biomedicine, Presidential Early Career Awards for Scientists and Engineers (PECASE), Robert Wood Johnson Policy Fellows, Searle Scholars, Sloan Research Fellows, Woodrow Wilson Fellows. As reported by the Top American Research Universities - see: http://mup.asu.edu/research data.html.

The number of National Academy members included in the National Academy of Sciences,

National Academy Members

Number of Post-Doctoral appointees

\section{Number of Science \& Engineering Disciplines nationally ranked in Top 100 for research expenditures}

\section{Return on Investment}

Total Research Expenditures (\$M)

\author{
Science \& Engineering \\ Research Expenditures in \\ non-medical/health \\ sciences
}

Percent of R\&D Expenditures
funded from External
Sources

\section{Patents Issued}

\section{Licenses/Options Executed}

\section{Licensing Income Received (\$M)}

\section{Number of Start-up Companies National rank is higher than predicted by Financial Resources Ranking based on US News \& World Report} National Academy of Engineering, and the Institute of Medicine. As reported by the Top American Research Universities - see: http://mup.asu.edu/research data.html.

As submitted to the National Science Foundation Survey of Graduate Students and Postdoctorates in Science \& Engineering (also known as the GSS) - see link.

The number of Science \& Engineering disciplines the university ranks in the top 100 (for public and private universities) based on the National Science Foundation's annual survey for R\&D expenditures, which identifies 8 broad disciplines within Science \& Engineering (Computer Science, Engineering, Environmental Science, Life Science, Mathematical Sciences, Physical Sciences, Psychology, and Social Sciences). Historically NSF provided these rankings (see tables 45-61 at link), but now data must be queried via WebCASPAR - see link.

Total expenditures for all research activities (including non-science and engineering activities) as reported in the National Science Foundation annual survey of Higher Education Research and Development (HERD).

This metric reports the Science \& Engineering total R\&D expenditures minus the research expenditures for medical sciences as reported by the National Science Foundation. Historically NSF provided these data (see link, table 36 minus table 52), but now data must be queried via WebCASPAR.

This metric reports the amount of research expenditures that was funded from federal, private industry and other (non-state and non-institutional) sources.

Source: National Science Foundation annual survey of Higher Education Research and Development (HERD).

The number of patents issued in the fiscal year as reported in the annual Accountability Report (table 6A).

Licenses/options executed in the fiscal year for all technologies as reported in the annual Accountability Report (table 6A).

License issue fees, payments under options, annual minimums, running royalties, termination payments, amount of equity received when cashed-in, and software and biological material end-user license fees of $\$ 1,000$ or more, but not research funding, patent expense reimbursement, valuation of equity not cashed-in, software and biological material end-user license fees of less than $\$ 1,000$, or trademark licensing royalties from university insignia. Data as reported in the annual Accountability Report (table 6A).

The number of start-up companies that were dependent upon the licensing of University technology for initiation as reported in the annual Accountability Report (table 6A).

This metric compares the overall national university ranking to the financial resources rank as reported by the US News and World report. 
Research Doctoral Degrees Awarded Professional Doctoral Degrees Awarded
The number of research doctoral degrees awarded annually as reported in the annual Accountability Report (table 5B).

The number of professional doctoral degrees awarded annually as reported in the annual Accountability Report (table 5B).

\section{Student Debt Summary}

\section{Percent of Bachelor's Recipients with Debt}

This is the percentage of bachelor's graduates in a given academic year who entered the university as a first-time-in-college (FTIC) student and who borrowed through any loan programs (institutional, state, Federal Perkins, Federal Stafford Subsidized and unsubsidized, private) that were certified by your institution - excludes parent loans. Source: Common Dataset (H4).

This is the average amount of cumulative principal borrowed (from any loan program certified by the institution) for each native, FTIC bachelor's recipient in a given academic year that graduated with debt - see metric definition above. This average does NOT include students who did not enter a loan program that was certified by the institution.

Source: Common Dataset (H5).

Student loan cohort default rate (CDR) data includes undergraduate and graduate students, and refers to the three federal fiscal year period when the borrower enters repayment and ends on the second fiscal year following the fiscal year in which the borrower entered repayment. Cohort default rates are based on the number of borrowers who enter repayment, not the number and type of loans that enter repayment. A borrower with multiple loans from the same school whose loans enter repayment during the same cohort fiscal year will be included in the formula only once for that cohort fiscal year. Default rate debt includes: Federal Stafford Loans, and Direct Stafford/Ford Loans - for more information see: http://ifap.ed.gov/DefaultManagement/CDRGuideMaster.html.

\begin{tabular}{|c|c|c|c|}
\hline \multicolumn{4}{|c|}{ Three Year CDR } \\
\hline $\begin{array}{l}\text { Cohort } \\
\text { Fiscal } \\
\text { Year }\end{array}$ & $\begin{array}{c}\text { Year } \\
\text { Published }\end{array}$ & $\begin{array}{l}\text { Borrowers in the Numerator } \\
\text { Borrowers in the Denominator }\end{array}$ & $\begin{array}{l}\text { 3-Yr Time Period } \\
\text { (Numerator) } \\
\text { 1-Yr Time Period } \\
\text { (Denominator) }\end{array}$ \\
\hline 2009 & 2012 & $\begin{array}{l}\text { Borrowers who entered repayment in } 2009 \\
\text { and defaulted in } 2009,2010 \text { or } 2011 \\
\text { Borrowers who entered repayment in } 2009\end{array}$ & $\frac{10 / 01 / 2008 \text { to } 9 / 30 / 2011}{10 / 01 / 2008 \text { to } 9 / 30 / 2009}$ \\
\hline 2010 & 2013 & $\begin{array}{l}\text { Borrowers who entered repayment in } 2010 \\
\text { and defaulted in } 2010,2011 \text { or } 2012 \\
\text { Borrowers who entered repayment in } 2010\end{array}$ & $\frac{10 / 01 / 2009 \text { to } 9 / 30 / 2012}{10 / 01 / 2009 \text { to } 9 / 30 / 2010}$ \\
\hline 2011 & $2014^{*}$ & $\begin{array}{l}\text { Borrowers who entered repayment in } 2011 \\
\text { and defaulted in } 2011,2012 \text { or } 2013 \\
\text { Borrowers who entered repayment in } 2011\end{array}$ & $\frac{10 / 01 / 2010 \text { to } 9 / 30 / 2013}{10 / 01 / 2010 \text { to } 9 / 30 / 2011}$ \\
\hline 2012 & 2015 & $\begin{array}{l}\text { Borrowers who entered repayment in } 2012 \\
\text { and defaulted in } 2012,2013 \text { or } 2014 \\
\text { Borrowers who entered repayment in } 2012\end{array}$ & $\frac{10 / 01 / 2011 \text { to } 9 / 30 / 2014}{10 / 01 / 2011 \text { to } 9 / 30 / 2012}$ \\
\hline 2013 & 2016 & $\begin{array}{l}\text { Borrowers who entered repayment in } 2013 \\
\text { and defaulted in } 2013,2014 \text { or } 2015 \\
\text { Borrowers who entered repayment in } 2013\end{array}$ & $\frac{10 / 01 / 2012 \text { to } 9 / 30 / 2015}{10 / 01 / 2012 \text { to } 9 / 30 / 2013}$ \\
\hline 2014 & 2017 & $\begin{array}{l}\text { Borrowers who entered repayment in } 2014 \\
\text { and defaulted in } 2014,2015 \text { or } 2016 \\
\text { Borrowers who entered repayment in } 2014\end{array}$ & $\frac{10 / 01 / 2013 \text { to } 9 / 30 / 2016}{10 / 01 / 2013 \text { to } 9 / 30 / 2014}$ \\
\hline 2015 & 2018 & $\begin{array}{l}\text { Borrowers who entered repayment in } 2015 \\
\text { and defaulted in } 2015,2016 \text { or } 2017 \\
\text { Borrowers who entered repayment in } 2015\end{array}$ & $\frac{10 / 01 / 2014 \text { to } 9 / 30 / 2017}{10 / 01 / 2014 \text { to } 9 / 30 / 2015}$ \\
\hline
\end{tabular}

